\title{
Original
}

\section{Simulation of Hydraulic Breaker Performance}

\section{-On Accumulator Behavior-}

\author{
by \\ Yoshio $_{\text {AKAHASHI }}{ }^{\dagger}$
}

\begin{abstract}
In this paper, using the analytical model and calculation procedure proposed by the author, the effects of gas volume and charged gas pressure in the accumulator on the performance of the large-sized hydraulic breaker have been studied, theoretically and experimentally. First, it is confirmed that the theoretical value of pressure drop ratio decreases with increase of the value of effective discharge gas volume inside the accumulator in case of the small-sized breaker, and correlates basically well with these experimental values. This is believed to indicate that these analytical model and method can analyze the accumulator behavior of an actual hydraulic breaker with extraordinary accuracy. In these simulation results, the percussion speed increases with increase of the value of the charged gas pressure within the range of calculation, while the mean supply flow rate decreases. Generally, it is considered that a proper value of the charged gas pressure inside the accumulator is $3.43 \mathrm{MPa}$. However, a value of the percussion speed is almost saturated with $\mathrm{Va}=0.3 \ell$. Accordingly, an appropriate value of gas volume inside the accumulator is 0.3 $\ell$, and also it should be determined by considering the size of a breaker.
\end{abstract}

Key Words : Hydraulic breaker, Accumulator, Performance, Simulation,

Design

\section{Introduction}

Hydraulic breakers are widely used in the fields of mining and public works as a machine for breaking rock and slag. In recent years, as the scale of projects has become larger, better performance and more durability have come to be demanded in this fields.

Uutil now, the author has done the performance tests using the same hydraulic breakers as used actualy at work sites in attempt to obtain the fundamental data for the design of hydraulic breakers. The author has also proposed an analytical model of the hydraulic breaker itself in order to evaluate its performance, and has proved that the performance of actual hydraulic breakers can be adequately evaluated through the comparative study on the experimental and theoretical values of

Received March 25, 1992

$\dagger$ Department of Mechanical Engineering, Mining College, Akita University, Akita City, Akita Prefecture 010, Japan 
the performance by using a numerical analysis applied to solve the proposed model.

In the previous papers, the effects of gas volume and charged gas pressure in the accumulator on the hydraulic breaker of small size were studied, theoretically and experimentally $[1,2]$.

In this paper, using the analytical model and the calculation procedure proposed by the author, simulations on the behavior of the largesized hydraulic breaker will be performed by solving analytically the hydraulic breaker model without using the numerical methods, and the effect of the accumulator behavior on the hydraulic breaker performance will be studied specially.

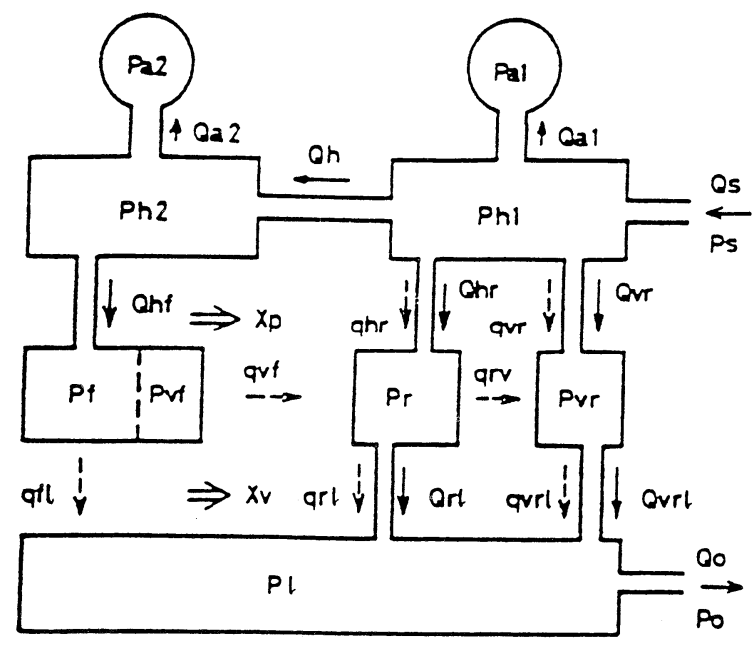

Fig. 1 Analytical model of hydraulic breaker

\section{Analytical Model}

The hydraulic breaker used in this simulation is assumed to be a type which breaks rock by the reciprocating motion to a piston which is provided by the mutually controlled actions of a free piston and a free valve. The piston strikes a chisel that is pressed against the object of the percussion, thereby propagaiting its striking energy in the chisel.

The analytical model shown in Figure 1 was conceived in order to analyse the performance of the hydraulic breaker. This figure shows the analytical model of the hydraulic breaker body only. In other words, this model comprises 7 pressure chambers and two accumulators, along with the oil supply port, discharge port and hydraulic lines.

The symbol $\mathrm{P}$ in the figure shows the pressure in a particular chamber and the various suffixes are denoted as follows: $\mathrm{Ph} 1$ and $\mathrm{Ph} 2$ are the pressures in high-pressure chamber 1, 2, respectively, $\mathrm{Pa}$ the pressure in accumulator, Pf and Pr the pressures in the piston fore-and rear-chambers, Pvf and Pvr the pressures in the valve fore-and rear-chambers, PI the pressure in the low-pressure chamber, Ps the supply pressure, and PO the discharge pressure. Here, the piston fore-chamber and the valve fore-chamber are the same pressure chamber. Hereinafter, these are referred to as the piston fore-chamber. The symbol $\mathrm{Q}$ shows the in/outlet flow rate of the oil inside the hydraulic lines, and $q$ shows the leakage flow rate. In addition, the cross-sectional areas of the piston which recieve the pressure are located in the piston fore-chamber and piston rear-chamber, and these areas thereof are represented by Af and Ar, respectively.

The valve has four cross-sectional areas which recieve the pressures in the piston fore-chamber, in the valve rear-chamber, in the low-pressure chamber and in the piston rear-chamber. The above mentioned these areas of the valve are represented by Avf, Avr, Al, Au, respectively. The symbols $\mathrm{Xp}$ and $\mathrm{Xv}$ in the figure represent the displacements of the piston and valve, respectively, and the directions of the arrows are positive. In addition, the point of impact is at $\mathrm{Xp}=\mathbf{0}$.

The flow rate $\mathrm{Q}$ and leakage flow rate $\mathrm{q}$ are defined respectively by the following equations. 


$$
\begin{aligned}
& \mathrm{Q}=\mathrm{G} \Delta \mathrm{P}, \mathrm{G}=\frac{\pi \mathrm{D}^{4}}{128 \rho \ell \ell} \\
& \mathrm{q}=\mathrm{g} \Delta \mathbf{P}, \mathrm{g}=\frac{\pi \mathrm{dh}^{3}}{12 \rho \nu \ell}
\end{aligned}
$$

Wherein, $G$ is the discharge coefficient of in/outlet oil flow between the chambers and $\mathrm{g}$ is the dischhrge coefficient of leakage flow. $\Delta \mathrm{P}$ is the pressure difference between the pressure chambers on either end of the line and $\mathrm{h}, \mathrm{d}, \mathrm{D}, \ell, \rho$ and $\nu$ are the clearance of the sliding surface, diameter of the piston, diameter of the hydraulic line, length of the hydraulic line, density of the hydraulic oil and kinematic viscosity of the hydraulic oil, respectively.

The hydraulic oil in this analytical model is assumed to be a incompressible viscous fluid, and the $\mathrm{Ni}$ gas in the accumlator is a ideal gas which is assumed to experience a polytropic change. Furthermore, it is assumed that the oil supply port is connected to a pump which is able to maintain a constant supply pressure, while the pressure in the discharge port is set at the atmospheric pressure $(\mathrm{P} 0=0.0981 \mathrm{MPa})$.

The hydraulic oil enters the hydraulic breaker from the oil supply port (Qs, Ps) and passes through the piston rear-chamber (Pr) and the valve rear-chamber (Pvr), respectively. Then, this oil passes through the low-pressure chamber $(\mathrm{Pl})$ and is discharged through the discharge port. The hydraulic oil is constantly supplied from a pump to the piston fore-chamber (Pf) through two highpressure chambers $(\mathrm{Ph} 1, \mathrm{Ph} 2)$. At this time, the accumulator acts as an auxiliary oil power source which supplements the hydraulic oil to the piston rear-chamber in the forward stroke of piston. By closing the line on the high-pressure side $(\mathrm{Ph} 1)$ of the piston rear-chamber and opening the line on its low-pressure side (P 1), the piston is caused to retreat. Next, the opposite condition causes to advance the piston and then the piston strikes a chisel. Both the line connecting the high-pressure chamber 1 with the piston rear-chamber and the line connecting the piston rear-chamber with the low-pressure chamber are opened and closed by means of the valve. Meanwhile, the lines at both ends of the valve rear-chamber, i. e., the line connecting the high-pressure chamber 1 whth the valve rear-chamber and the line connecting the valve rear- chamber with the low-pressure chamber, are opened and closed by the piston which has a reciprocating motion in the valve. Thus, the piston and valve are controlled mutually.

In this analytical model, it is believed, e. g., that the closure of the line that connects the highpressure chamber 1 with the piston rear-chamber and of the line that connects the piston rearchamber with the low-pressure chamber generates leakage of the pressure oil, and that there is constant leakage from the sliding components.

Three types of equations are obtained from this analytical model : viz., the epuation of continuity, the equations of the accumulator state, and the equations of motion for the piston and valve.

The analytical model used in this analysis is fundamentally the same as the analytical model proposed preriously by the author. However, in this analytical model, the lower-pressure accumulator and static friction resistance of the piston, which have virtually no effect on the behavior of the system, have been omitted. In addition, the behavior of the gas inside the accumulators has been linearized in this model in order to obtain an analytical solution by using a linear equation of state with a constant coefficient. 


\section{Theoretical equations}

\section{1 Equations of continuity}

The equations of continuity for 6 pressure chambers in Figure 1 are shown in Equation (2).

High-pressure chamber $1 \quad \boldsymbol{Q} s=\boldsymbol{Q} a_{1}+Q \boldsymbol{Q}+\hat{\mathbf{Q}} \boldsymbol{h} r+\hat{\mathbf{Q}} v \boldsymbol{r}$

High-pressure chamber $2 \quad \boldsymbol{Q h}=\boldsymbol{Q} \boldsymbol{a}_{2}+\mathbf{Q h f}$

Low-pressure chamber $\quad q f l+Q r l+\hat{Q} v r l+A v l \frac{d X p}{d t}+Q o$

Piston fore-chamber $\quad Q h f=q v f+q f l+A f \frac{d X p}{d t}=A v f \frac{d X v}{d t}$

Piston rear-chamber

$$
\hat{Q} h r+q v f+A r \frac{d X p}{d t}=q r v+\hat{Q} r l+A u \frac{d X v}{d t}
$$

Valve rear-chamber

$$
\hat{Q} v r+q r v+A v r \frac{d X v}{d t}=\hat{Q} v r l
$$

Wherein, the symbol $\sim$ shows the quantity that changes depending on the positions of the piston and valve. When Equations (1) is substituted into Equation (2) and expressed in a matrix form, it becomes as follows :

$$
\widetilde{G}\left[\frac{w}{u}\right]=[\widetilde{R} \mid 0]\left[\frac{\mathrm{x}}{\mathrm{z}}\right]+[0 \mid \widetilde{S}]\left[\frac{\dot{x}}{z}\right]+\widetilde{T} P
$$

where,

$$
\widetilde{G}=\left[\begin{array}{ccc}
G s+G h+\hat{G} h r+\hat{G} v r & \\
-G h & G h+G h f & \\
0 & 0 & G o+\hat{G} r l+\hat{G} v r l+g f l \\
0 & -G h f & -g f l \\
-\hat{G} h r & 0 & -\hat{G} r l \\
-\hat{G} v r & 0 & -\hat{G} v r l
\end{array}\right.
$$

\section{Symmetric}

$$
\begin{gathered}
\begin{array}{c}
G h f+g f l+g v f \\
-g v f \\
0
\end{array} \\
\widetilde{R}=\left[\begin{array}{cccc}
0 & 0 & 0 & 0 \\
0 & 0 & 0 & 0 \\
0 & 0 & 0 & -A v l \\
0 & 0 & -A f & -A v f \\
0 & 0 & A r & -A v \\
0 & 0 & 0 & A v r
\end{array}\right], \quad \widetilde{T}=\left[\begin{array}{ccc}
G s & 0 \\
0 & 0 \\
0 & G o \\
0 & 0 \\
0 & 0 \\
0 & 0
\end{array}\right], \quad \widetilde{S}=\left[\begin{array}{ll}
1 & 0 \\
0 & 1 \\
0 & 0 \\
0 & 0 \\
0 & 0 \\
0 & 0
\end{array}\right]
\end{gathered}
$$




$$
w^{*}=\left[\frac{w}{u}\right]=\left[\begin{array}{c}
P h_{1} \\
P h_{2} \\
P l \\
\frac{P f}{P r} \\
P v r
\end{array}\right], \quad x^{*}=\left[\frac{x}{z}\right]=\left[\begin{array}{c}
X p \\
X v \\
\dot{X} p \\
\dot{X} v \\
\hline \delta V a_{1} \\
\delta V a_{2}
\end{array}\right], \quad p=\left[\begin{array}{l}
P s \\
P o
\end{array}\right], \quad \dot{x}^{*}=\frac{d}{d t}\left[\frac{x}{z}\right]=\left[\frac{\dot{x}}{z}\right]
$$

where, $\widetilde{G}, \widetilde{R}, \widetilde{T}$ and $\widetilde{S}$ are invariable matrices, and $w^{*}$ and $x^{*}$ are variable vectors and $\mathrm{p}$ is a constant vector. $\delta V a_{1}$ and $\delta V a_{2}$ in the above equation are the very small volume fluctuations of the gases in the two accumulators, and are defined as shown in Equation (4).

$$
\begin{aligned}
& \boldsymbol{Q} \boldsymbol{a}_{1}=-\delta \boldsymbol{V} \boldsymbol{a}_{1} \\
& \boldsymbol{Q} \boldsymbol{a}_{2}=-\delta \boldsymbol{V} \boldsymbol{a}_{2}
\end{aligned}
$$

The following equation is obtained by transforming Equation (3).

$$
w^{*}=[R \mid 0] x^{*}+[0 \mid S] \dot{x}^{*}+T p
$$

where,

$$
R=\widetilde{G}^{-1} \widetilde{R}, S=\widetilde{G}^{-1} \widetilde{S}, T=\widetilde{G}^{-1} \widetilde{T}
$$

where, R, S and T are constant matrices. Equation (5) shows that the pressure of each chamber $w^{*}$ can be expressed as the sum of $\boldsymbol{x}^{*}, \dot{x}^{*}$ and $\mathbf{p}$.

3. 2 Equations of motion for the piston and valve

The equation of motion for the piston is given by equation (6).

$$
M p \frac{d \dot{X} p}{d t}=A f P f-A r P r-C p \dot{X} p
$$

where, $\mathrm{Mp}$ is the piston mass and $\mathrm{Cp}$ is the viscous resistance coefficient of the piston.

On the other hand, the equation of motion for the valve is shown in Equation (7).

$$
\begin{aligned}
M v \frac{\mathrm{d} \dot{\mathrm{X}} \mathbf{v}}{\mathrm{dt}}=A v l P l & +A v f P f+A u P r \\
& -A v r P v r-C v \dot{X} v-\rho \hat{Q} h r v \cos \phi
\end{aligned}
$$

where, $\mathrm{Mv}$ is the valve mass, $\mathrm{Cv}$ is its viscous resistance coefficient, $\mathrm{v}$ is the flow velocity passing through the orifice and $\phi$ is the inlet flow angle, which is set at $\phi=69^{\circ}$. The sixth term on the righthand side of Equation (7) shows the flow force generated by the flow rate from the high-pressure chamber 1 into the piston rear-chamber. This flow force can be calculated by using Equation (8).

$$
\rho \hat{\mathbf{Q}} h r v \cos \phi=2 c \cdot c \mathrm{v} \cdot \boldsymbol{a} \cdot \cos \phi\left(P h_{1}-P r\right)
$$

where, $\mathrm{C}$ is the flow rate constant, $\mathrm{cv}$ is the velocity coefficient and $\boldsymbol{a}$ is the cross-sectional area of the orifice opening. When the equations of motion for the piston and valve are combined and made into a vector matrix expression, the following simultaneous ordinary differential equation of the first order is obtained.

$$
\dot{x}=A x+B u+D w
$$

where,

$$
A=\left[\begin{array}{cccc}
0 & 0 & 1 & 0 \\
0 & 0 & 0 & 1 \\
0 & 0 & -C p / M p & 0 \\
0 & 0 & 0 & -C v / M v
\end{array}\right]
$$




$$
\begin{aligned}
B & =\left[\begin{array}{cccc}
0 & 0 & 0 \\
0 & 0 & 0 \\
A f / M p & -A r / M p & 0 \\
A v f / M v & (A v+2 c \cdot c \mathrm{v} \cdot a \cos \psi) / M v & -A v r / M v
\end{array}\right] \\
D & =\left[\begin{array}{cccc}
0 & 0 & 0 & -2 c \cdot c v \cdot a \cos \psi / M v \\
0 & 0 & 0 & 0 \\
0 & 0 & 0 & A v l / M v
\end{array}\right] T
\end{aligned}
$$

\section{3 Equation of state for the gas inside the accumulators}

Politropic change is assumed as the behavior of the gas inside the accumlators.

$$
\begin{aligned}
& P a_{i} V a_{i}{ }^{\mathrm{n}}=\text { Const. } \\
& \dot{V} a_{i}=-G a\left(P h_{i}-P a_{i}\right)
\end{aligned}
$$

where, $\mathrm{n}$ is the politropic index, and in this paper, $n=k$ ( $k$ : adiabatic index). Pai represents the gas pressure, Vai represents the gas volume, Ga represents the flow rate constant, and Phi represents the pressure in the high-pressure chamber. In order to find the analytical solution, the linearisation of Eguation (10) must be considered. If the very small volume fluctuations $\delta V a_{i}$ is considered from the reference state expressed as Pai $(0)=$ Pair and Vai $(0)=$ Vair, then Vai is expressed by the following equation.

$$
V a_{i}=V a_{i} r+\delta V a_{i}
$$

From the above equations, if Taylor series are expanded around the reference gas volume Vaio and only the primary term is taken, it becomes the following equation.

$$
\delta \dot{V} a_{i}=-G a n \frac{P a_{i} r}{V a_{i} r}-G a\left(P h_{i}-P a_{i} r\right)
$$

When Equation (13) is expressed by the vector matrix for the accumulators, it becomes Equation (14).

$$
\dot{z}=F z+G w+H y
$$

where,

$$
\begin{aligned}
& F=\left[\begin{array}{cc}
-G a_{1} n \frac{P a_{1} r}{V a_{1} r} & 0 \\
0 & -G a_{2} n \frac{P a_{2} r}{V a_{2} r}
\end{array}\right] \\
& G=\left[\begin{array}{ccc}
-G a_{1} & 0 & 0 \\
0 & -G a_{2} & 0
\end{array}\right], \quad H=\left[\begin{array}{cc}
G a_{1} & 0 \\
0 & G a_{2}
\end{array}\right] \\
& y=\left[\frac{P a_{1} r}{P a_{2} r}\right], \quad z=\left[\frac{\delta V a_{1}}{\delta V a_{2}}\right], \quad w=\left[\begin{array}{c}
P h_{1} \\
P h_{2} \\
P 1
\end{array}\right]
\end{aligned}
$$

\section{4 Equations of state and output equations}

The following two equations are obtained by combining Equations (5), (9) and (14).

$$
\dot{x}^{*}=E^{*} x^{*}+J^{*} p^{*}
$$




$$
\boldsymbol{w}^{*}=\boldsymbol{M}^{*} \boldsymbol{x}^{*}+\boldsymbol{U}^{*} \boldsymbol{p}^{*}
$$

where,

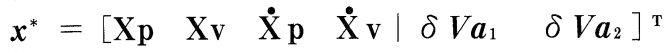

$$
\begin{aligned}
& \boldsymbol{w}^{*}=\left[\begin{array}{lllll}
P h_{1} & P h_{2} & P l \mid P f & P r & P v r
\end{array}\right]^{\mathrm{T}}
\end{aligned}
$$

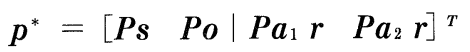

$$
\begin{aligned}
& \begin{array}{ll}
E^{*} & =\left[I-B^{*} S^{*}\right]^{-1}\left[A^{*}+B^{*} R^{*}\right] \\
J^{*} & =\left[I-B^{*} S^{*}\right]^{-1}\left[R^{*}+S^{*} A^{*}\right]
\end{array} \quad D^{*}=\left[\frac{0}{H}\right] \\
& M^{*}=\left[I-B^{*} S^{*}\right]^{-1}\left[R^{*}+S^{*} A^{*}\right], \quad S^{*}=[0 \mid S] \\
& U^{*}=\left[I-B^{*} S^{*}\right]^{-1}\left[S^{*} D^{*} \mid T\right], R^{*}=[R \mid 0] \\
& A^{*}=\left[\begin{array}{c|c}
A & \mathbf{0} \\
\hline \mathbf{0} & \boldsymbol{F}
\end{array}\right], \quad B^{*}=\left[\begin{array}{c|c}
\boldsymbol{D} & \boldsymbol{B} \\
\hline \boldsymbol{G} & \mathbf{0}
\end{array}\right]
\end{aligned}
$$

Equation (15) is a linear simultaneous ordinary differential equation, normally called the equation of state .

The $x^{*}$ vector is the called the state vector, which comprises the displacements and velocities of the piston and valve and very small fluctuation in the gas volume inside the accumulators, and is a quantity which changes depending on time.

The $p^{*}$ vector is input vector, which comprises the supply pressure, discharge port pressure and the reference gas pressure for the two accumulators, and is assumed to maintain a constant value, regardless of time.

Equation (16) is a simultaneous equation which is normally referred to as the output equation. The $w^{*}$ vector is called the output vector, which comprises the pressures in the various chambers, and is expressed as the linear combination between the state vector and the input vector. $E^{*}, J^{*}$, $M^{*}$ and $U^{*}$ are constant matrices which change depending on the switching of the valve, the piston displacement and the changes in pressure, otherwise they are constant in relation to time.

The above two equations construct the state model for this analytical model.

\section{5 Method for solving the equation of state}

As discussed above, the various coefficient matrices of the equation of state change depending on the switching of the valve, etc., creating a variable coefficient system, but by subdividing the system into partial systems at the switching point, the system can be regarded as linear constantcoefficient systems within the partial segments, for which analytical solutions exist. The analytical solution of the equation of state, Equation (15), is shown in the following equation.

$$
x^{*}(t)=e^{E * t} x^{*}(0)+\int_{0}^{t} e^{E * t} J^{*} p^{*} d \tau
$$

The first term on the righthand side of Equation (17) is a transient term and second is a forced term. Wherein, $e^{E * t}$ is called the transitive matrix and expressed as shown below.

$$
e^{E * t}=L^{-1}\left\{\left[s I-E^{*}\right]^{-1}\right\}=\sum_{i=0}^{\infty} \frac{1}{i !} E^{* i} t^{i}
$$

Provided that $L^{-1}\{\}$ is defined as the inverse Laplace transformation and $\mathrm{s}$ as the Laplace operator. The infinite series expression of Equation (18) can also be rewritten as another finite series expression, by which the computations are simplified.

In this analysis, the degree of $E^{*}$ is 6 . Using various practical design parameters (dimensions of parts, such as Af and Ar, etc.) and combining the eigenvalues for the constant matrices $E^{*}$ for 
each individual partial system, the analytical solution was found in the types of the 4 following equations.

$$
\begin{aligned}
& e^{E^{*} t}=\sum_{j=1}^{k} R_{j} t^{j-1}+\sum_{j=\mathrm{k}+1}^{6} R_{j} e^{\lambda j t} \\
& x^{*}(t)=e^{E^{*} t} x(0)+\left[\sum_{j=1}^{k} R_{j} \frac{1}{j} t^{j}+\sum_{j=\mathrm{k}+1}^{6} \frac{R_{j}}{\lambda_{j}}\left(e^{\lambda j t}-1\right)\right] J^{*} p^{*}
\end{aligned}
$$

Type $1: \mathbf{k}=3, \lambda_{\mathrm{j}}(\mathrm{j}=1$ through $\mathrm{k}$; an equal root of $\mathbf{0}$,

$$
\mathrm{j}=\mathrm{k}+1 \text { through } 6 ; 3 \text { different real roots) }
$$

Type $2: \mathrm{k}=2, \lambda_{\mathrm{j}}(\mathrm{j}=1$ through $\mathrm{k} ;$ an equal root of 0 ,

$$
\begin{aligned}
& \mathrm{j}=\mathrm{k}+1 \text { through } 6 ; 4 \text { different real roots }) \\
& \boldsymbol{e}^{E^{*} t}=\sum_{j=1}^{k} \boldsymbol{R}_{j} \boldsymbol{t}^{j-1}+\sum_{j=\mathrm{k}+1}^{4} \boldsymbol{R}_{j} \boldsymbol{e}^{\lambda j t}+\boldsymbol{R}_{5} \boldsymbol{e}^{a t} \cos \beta \mathrm{t}+\boldsymbol{R}_{6} \boldsymbol{e}^{a t} \sin \beta \mathrm{t} \\
& \boldsymbol{x}^{*}(\mathrm{t})=\boldsymbol{e}^{E * t} \boldsymbol{x}^{*}(\mathbf{0})+\left[\sum_{j=1}^{4} \boldsymbol{R}_{j} \frac{1}{j} t^{j}+\sum_{j=k+1}^{4} \frac{\boldsymbol{R}_{j}}{\lambda_{j}}\left(\boldsymbol{e}^{\lambda_{j} t}-1\right)\right. \\
& +\frac{\boldsymbol{R}_{5}}{\alpha^{2}+\beta^{2}}\left\{-\alpha+\boldsymbol{e}^{a t}(\alpha \cos \beta \boldsymbol{t}+\beta \sin \beta \boldsymbol{t})\right\} \\
& \quad+\frac{\boldsymbol{R}_{6}}{\alpha^{2}+\beta^{2}}\left\{\beta+\boldsymbol{e}^{a t}(\alpha \sin \beta \boldsymbol{t}-\beta \cos \beta \boldsymbol{t}\}\right] \boldsymbol{J}^{*} \boldsymbol{p}^{*}
\end{aligned}
$$

Type $3: \mathrm{k}=3, \lambda_{\mathrm{j}}(\mathrm{j}=1$ through $\mathrm{k}$; an equal root of 0 ,

$$
\begin{aligned}
& \mathrm{j}=\mathrm{k}+1 \text { through } 4 ; \text { a real root, } \\
& \mathrm{j}=5,6 ; \text { a pair of complex root, } \alpha \pm i \beta)
\end{aligned}
$$

Type $4: k=2, \lambda_{j}(j=1$ through $k$; an equal root of 0 ,

$$
\begin{aligned}
& \mathrm{j}=\mathrm{k}+1 \text { through } 4 ; 2 \text { a real roots, } \\
& \mathrm{j}=5,6 ; \text { a pair of complex root, } \alpha \pm i \beta)
\end{aligned}
$$

Wherein, $R_{j}(j=1, \cdots, 6)$ is the constant-coefficient matrix and $x^{*}(0)$ is initial value for $x^{*}(t)$ when $\mathrm{t}=\mathbf{0}$.

The analytical solutions for all of the partial systems are in the types of the 4 equations, and the system as a whole is constructed by the combination thereof.

\section{6 Method for computing characteristic values through analytical solution}

The charactlristic values for a hydraulic breaker can be computed by using the equation of state derived in the above mentioned manner. However, these characteristic values change when crushing a rock in case of an actual hydraulic breaker, because of the repelling force from the object hit by the breaker percussion. Therefore, this repelling force must be considered in the about-mentioned calculations.

Thus, the repelling force of the percussion object was taken into consideration in this analysis, and computations were performed by assuming the coefficient of repellent. However, regarding the velve, analysis was performed without assuming the coefficient of repellent, since the stroke of the valve was very short compared with that of the piston.

The dimensions of parts for an actual hydraulic breaker were used in the case of these calculations.

\section{RIsults and Considerations}

Accumulator is intended to store pressurized fluid for the time when a system needs an additional supply in a hurry. 
Figure 2 shows a comparison between the theoretical and experimental values of pressure drop ratio ( $\Delta \mathrm{P} /$ Pgmax : $\Delta \mathrm{P}=$ Pgmax - Pgmin, Pgmax and Pgmin are the maximum and minimum values of pressure inside the accumulator in the forward stroke of piston) inside the accumulator in case of the small-sized breaker. $\Delta \mathrm{P} /$ Pgmax decreases with increase of the values of effective discharge gas volume ( $\Delta \mathrm{V}: \Delta \mathrm{V}=\mathrm{V} \max -\mathrm{V} \min , \mathrm{Vmax}$ and $\mathrm{Vmin}$ are the maximum and minimum values of gas volume inside the accumulator in the forward stroke of piston) inside the accumulator.

According to the figure, the theoretical values of $\Delta \mathrm{P} /$ Pgmax correlates basically well with the experimental values.

This phenomenon indicate that the analytical model and method used in this study can analyze the accumulator behavior of an actual hydraulic breaker with extraordinary accuracy.

On basis of these results, a simulation was studied on the accumulator behavior in the case of the large-sized breaker. In this case, only the values of charged gas pressure and gas volume inside the accumulator were changed, and the crosssectional area of the piston fore- (Af) and rear(Ar) chambers were increased by two or three times compared with those of the small-sized breaker.

Figure 3 and 4 show the effect of the values of charged gas pressure $(\mathrm{Pg})$ inside the accumula-

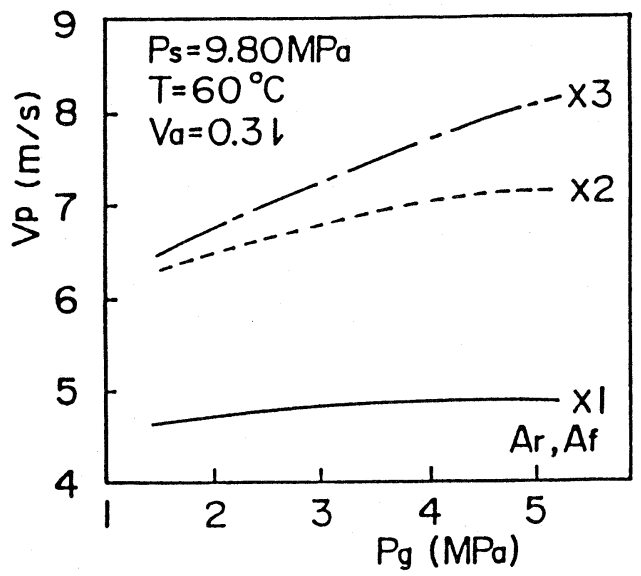

Fig. 3 Effect of values of charged gas pressure inside accumulator on percussion speed.

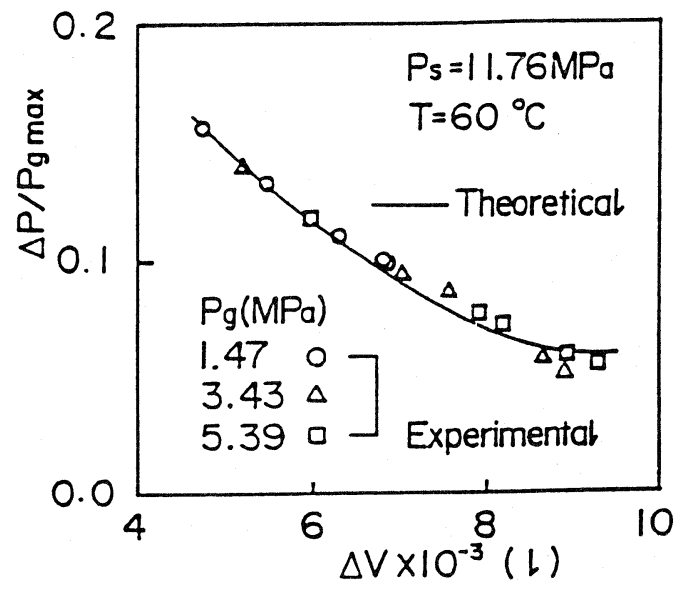

Fig. 2 Relationship between values of pressure drop ratio and values of effective discharge gas volume inside accumulator in piston forward stroke.

(in the case of the small-sized breaker)

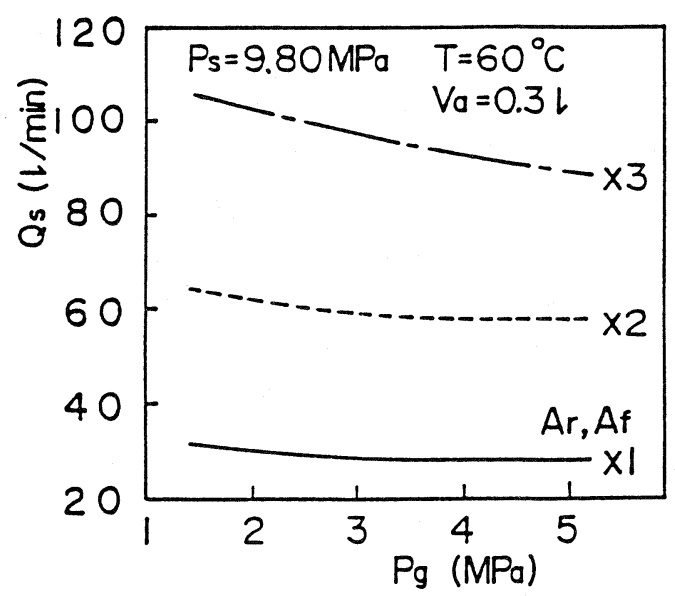

Fig. 4 Effect of values of charged gas pressure inside accumulator on mean supply flow rate. 


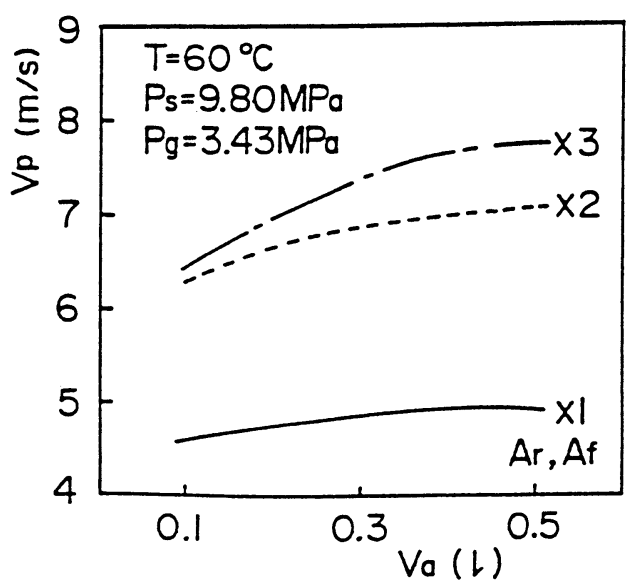

Fig. 5 Effect of values of gas volume inside accumulator on percussion speed.

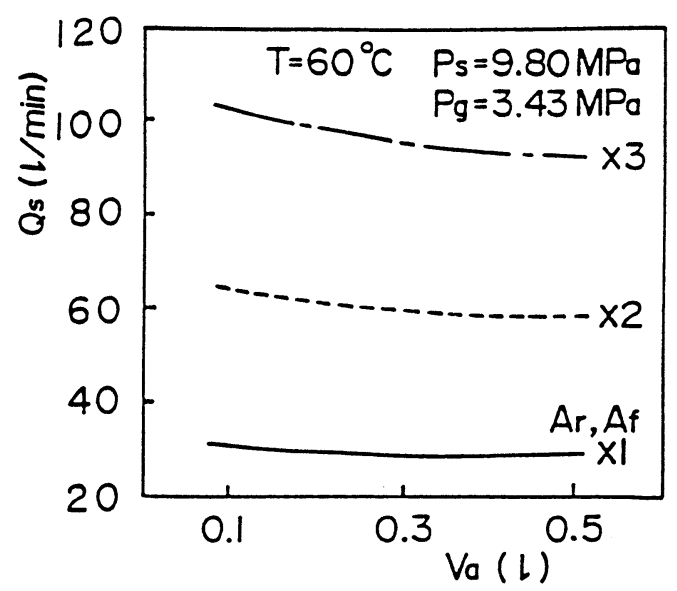

Fig. 6 Effect of values of gas volume inside accumulator on mean supply flow rate.

tor on the percussion speed $(\mathrm{Vp})$ and the mean supply flow rate $(\mathrm{Qs})$, respectively. The percussion speed increases with increase of the values of the charged gas pressure within the range of calculating, while the mean supply flow rate decreases. Generally, it is considered that a proper value of the charged gas pressure inside the accumulator is 3.43 $\mathrm{MPa}$.

Figure 5 and 6 show the effect of the values of gas volume ( $\mathrm{Va}$ ) inside the accumulator on the percussion speed and the mean supply flow rate, respectively. These figures show a similar tendency in Figure 3 and 4. However, the value of the percussion speed is almost saturated at $\mathrm{Va}=0.3 \ell$. As a result of these figures, an appropriate value of gas volume inside the accumulator is $0.3 \ell$, and also it should be determined by considering the size of a breaker.

\section{Conclusion}

On designing a hydraulic breaker, the analysis of its performance for various design parameters can be easily performed by using the analytial techniques shown in this study.

\section{References}

1) Y. Takahashi, Y. Shibuya, G. Obinata, T. Nakamura and S. Kizawa, J. of the Mining and Materials Processing Institute of Japan, 106 No. 2 (1990) 77.

2) Y. Takahashi, Y.Shibuya, G. Obinata, T. Nakamura and S. Kizawa, J. of the Mining and Materials Processing Institute of Japan, 106 No. 1 (1990) 35. 